\title{
Gait Quantification and Visualization for Digital Healthcare
}

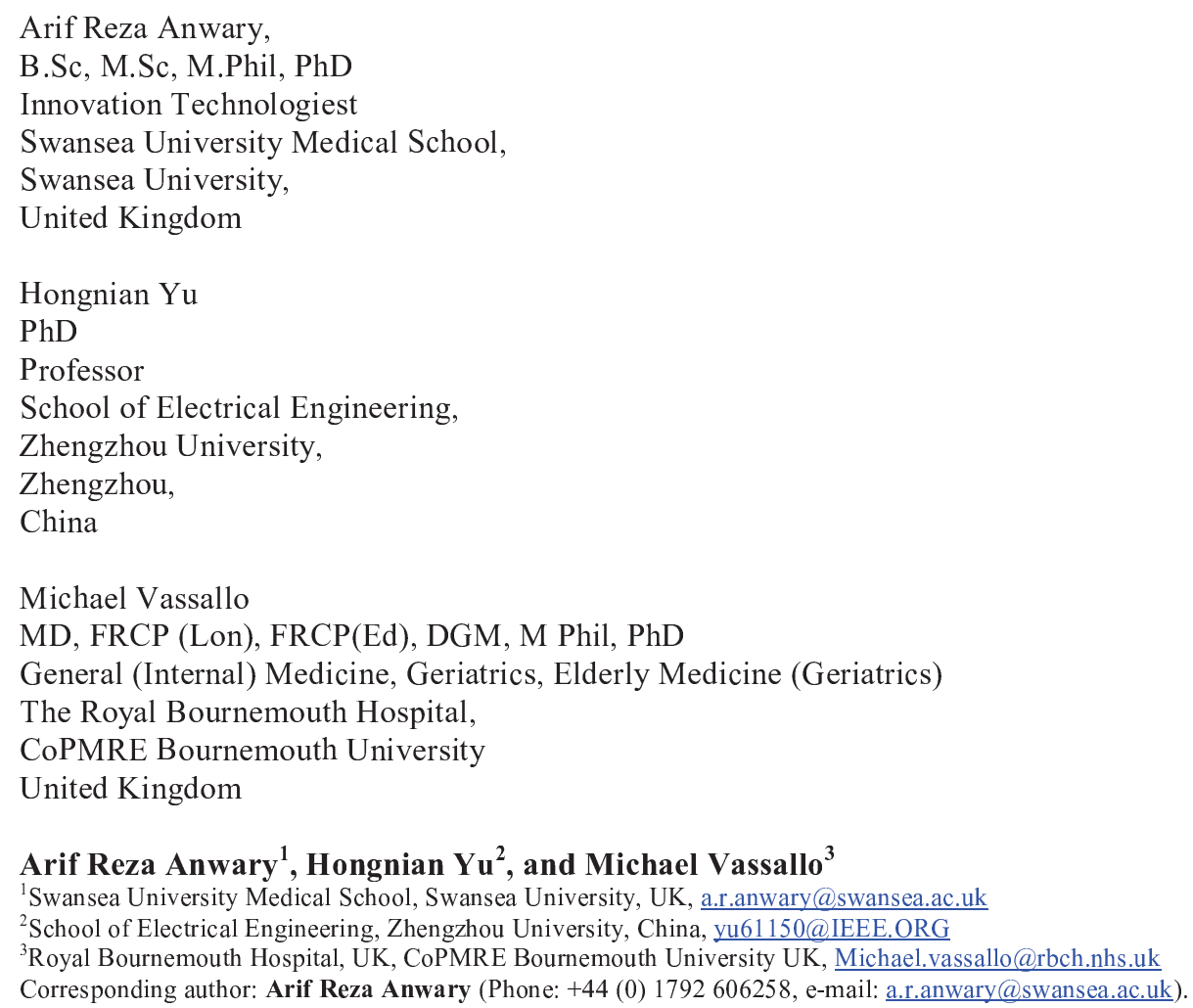

Funding: This work was supported in part by the Erasmus Mundus Fusion Project under Grant 545831-EM-1-20131-IT-ERAMUNDUSEMA21, in part by the European Commission Marie Skłodowska-Curie SMOOTH (Smart Robots for Firefighting) Project under Grant H2020-MSCA-RISE-2016-734875 and CHARMED project (H2020MSCA-RISE-2016-734684, and in part by the Royal Society International Exchanges Scheme (Adaptive Learning Control of a Cardiovascular Robot Using Expert Surgeon Techniques) Project under Grant IE151224. The authors would like to thank all subjects that participated in the study.

\section{Competing interests: None declared}

Ethical approval: Ethical approval for this research was granted by the Bournemouth University ethical review committee and each subject was given a Participant Information Sheet and signed an informed Participant Agreement Form. 
Acknowledgement: Authors would like to thank all subject participated in this study.

Keywords: Gait Visualization, Gait Asymmetry, Gait Feature Extraction, Inertial Measurement Unit, Gait Analysis.

A running title: Gait Quantification and Visualization 


\title{
Gait Quantification and Visualization for Digital Healthcare
}

\begin{abstract}
Gait abnormalities are common in clinical practice and there is a global imperative to improve technologies that facilitate their detection, evaluation, monitoring and management. Real time evaluation using digital technology supports the development of digital healthcare. Currently gait assessment relies on visual observation of structured clinical tests such as the "Timed Get up and Go Test". Gold standard methods such as "Qualisys Motion Capture System" require sophisticated equipment in gait laboratories. These are not widely available due to expense, analysis time and requirement of trained technicians. Developing low cost, portable, easy to use digital technology is important to enable sophisticated assessment of gait at home or in clinics. Common measures for quantification of gait include symmetry angle, ratio and index. These measurements may be difficult to interpret by users as standalone values. To facilitate the evaluation and interpretation of locomotive information, a tool to visualize gait in real-time is proposed. The proposed tool consists of five approaches (1: Real-time dial visualization, 2: Visualization of individual leg time variation, 3: Visualization of both legs asymmetry, 4: Boxplot visualization, and 5: Evaluation considering all features). Results show that wearable Inertial Measurement Unit (IMU) can be used for extraction of objective gait features. This system opens possibilities for home-based assessment of gait without the requirement and expense of an elaborate laboratory setup and supports the development of digital healthcare.
\end{abstract}

\section{Keywords}

Gait Visualization, Gait Asymmetry, Gait Feature Extraction, Inertial Measurement Unit, Gait Analysis.

\section{Introduction}

A major challenge for researchers and clinicians who address healthcare issues in the ageing population, is to monitor functioning, and to timely initiate interventions that aim to prevent loss of functional abilities to improve the quality of life of older people (1). Gait change is an indicator of different diseases and disease progression and has also been shown to be a determinant of recovery in patients with various medical conditions such as stroke (2) and Parkinson's disease (3). It has also been used to monitor patient progress in orthopaedics and rehabilitation (4). It is also a predictor of functional and cognitive decline (5) and results in a reduced quality of life. Gait changes have therefore attracted the interest of researchers in various disciplines and its measurement is of importance. 
Digital healthcare has revolutionized business models in a variety of industries, however, the adoption of digital services in healthcare has progressed at a relatively slow pace. Therefore, the need for more profound transformation in healthcare systems has intensified in recent years due to social needs and technological developments (6). In current clinical practice, validated clinical tests are mostly used to identify gait abnormalities. Clinical scales used to analyze gait parameters are subjective or semi-subjective and a poor replacement to laboratory-based methods for identifying changes in gait patterns such as symmetry and balance. There are several assessment tools for this purpose such as the the Gait Abnormality Rating Scale (7), Figure of 8 Walk Test (8), and Berg Balance Scale (9). These are mostly based on visual observation, rely on clinical expertise for scoring and often abnormalities are described in a crude categorical way as being present or not. More sophisticated analysis of gait requires measures such as thee Symmetry index (SI) (10), Symmetry ratio (SR) (11), Ratio (Ia) (12), Gait asymmetry (GA) (13), Symmetry angle (SA) (14) and Symmetry indices (15). However this leads to difficulties how to interpret results and what constitutes normal gait particularly for younger and older individuals. The most commonly used SI approach is to normalize to a reference value (16) has the potential for error as gait changes with age (17). A mean value calculation used for quantifying gait asymmetry may also lead to erroneous results as the mean measurements from two abnormal limbs may appear normal. There are visualization techniques of hand gestures (18, 19), motion gesture (20), handheld devices (21) and complex activities (22). These techniques are applied for detecting simple gestures to complex activities. We will explore these in our future work. Another visual representation method of examining asymmetric gait was developed (23). Here gait changes are presented as pattern deviations relative to normative data by color-coding the magnitude and the direction of the deviation. This system lacks quantitative information and does not examine changes in symmetry of bilateral parameters. Currently such detailed analysis of gait depends on expensive equipment based in gait labs. This, makes it financially unaffordable to the majority of patients. It also requires patients to travel to the site where the equipment is based and therefore is not suitable for patients who have difficulty in travelling. New affordable and portable products are therefore needed to increase the availability to all patients and reduce health inequalities. There is a need for the transformation of gait assessment from a hospital or laboratory centered system to a person or home centered environment (6). An important aspect of any new developments is a cost benefit analysis to ensure that any new developments offer good value for money (24). 
To address these considerations, we develop a simple, affordable and wearable multi-IMU based user friendly gait visualization tool which is easy to interpret results. The proposed tool consists of five visualization approaches that provide the comprehensive assessment of gait: 1) Real time visualization; 2) Visualization of individual leg time variation; 3) Visualization of both legs asymmetry; 4) Boxplot based visualization; and 5) Gait evaluation considering all features. It is portable to make it usable in routine clinical practice including the patient's home. To date, an automatic real time gait visualization technique based on affordable fused synchronous accelerometer and gyroscope data is not commercially available. The aim of this study is to visualize gait information based on accelerometer and gyroscope data to increase the reliability and validity of monitoring gait abnormalities using the automatic lower limb gait features extraction method proposed by Anwary AR et al (25). This can support digital healthcare through the development and use of new and affordable technologies for diagnosis and monitoring of gait that results in structural change in the delivery of healthcare that can be delivered in the patient's own home (24).

Next section presents our proposed method. This includes the data collection, gait feature extraction, visualization of gait. Result section delivers the experimental results to demonstrate the proposed method. Next section is discussions section and last section is conclusion.

\section{Methodology}

The materials and methods used for the development of this work are described in the following subsections.

\section{Data collection}

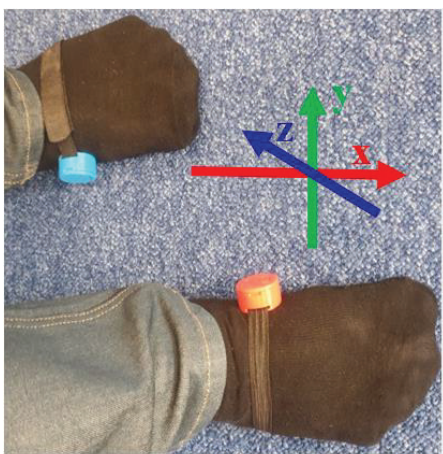

(a)

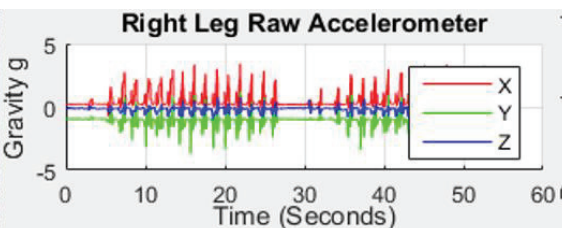

Left Leg Raw Accelerometer

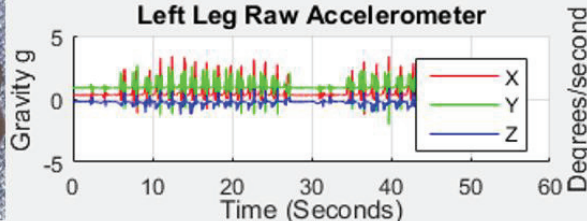

(b)

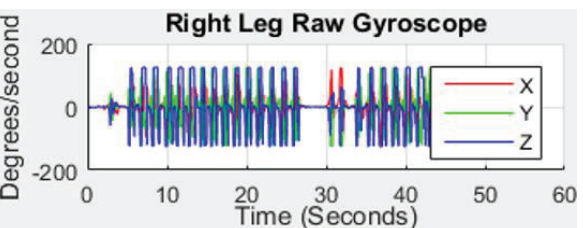

Left Leg Raw Gyroscope

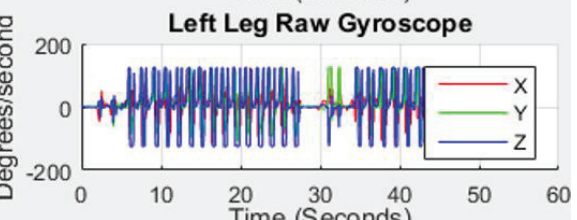


Figure 1. (a) IMU sensors placement in right and left metatarsal feet locations, and (b) Raw accelerometer

\section{and gyroscope data of young subject 1}

Sensors are placed at metatarsal foot locations of both legs for collecting data since this can achieve the best performance (25). We design and develop an android app for synchronous data collection from accelerator and gyroscope (26). A convenience sample of 20 subjects is recruited with 10 healthy young subjects and 10 older subjects. Their demographic details are shown in Table 1.

Table 1: Demographic statistics (mean \pm standard deviation) of the subjects

\begin{tabular}{|c|c|c|}
\hline & Group 1: Young & Group 2: Older \\
\hline Number & 10 & 10 \\
\hline Gender (Male/Female) & $9 \mathrm{M}, 1 \mathrm{~F}$ & $9 \mathrm{M}, 1 \mathrm{~F}$ \\
\hline Age & $25.3 \pm 4.64$, range 19-35 years & $69.4 \pm 7.28$, range 62-86 years \\
\hline Height $(\mathrm{cm})$ & $161.4 \pm 16.9$ & $152.3 \pm 15.4$ \\
\hline Weight $(\mathrm{kg})$ & $61.9 \pm 4.61$ & $63.4 \pm 3.03$ \\
\hline Body mass index & $24.45 \pm 5.77$ & $28.14 \pm 6.07$ \\
\hline
\end{tabular}

Subjects perform a walk in a straight corridor comprising of 15 strides of normal forward walking, a turn-around and another 15 strides. Accelerometer and gyroscope data are collected by placing the sensors on right and left metatarsal foot locations of the barefoot. Accelerometer and gyroscope raw data for all the strides from young subject 1 is presented in Figure 1(b). Our method has been validated to pick up regular features occurring in the human gait cycle (stride) (26) that has the salient features.

\section{Gait features extraction}

\section{Stride, stance, swing and step phase detection}

The stride detection technique is used based on the local minimal prominence characteristics of strides associated with the time-varying magnitude of acceleration (26). This detected the foot's initial contact to the ground (start of gait cycle), the end of the stance and the beginning of the swing phase and the end of swing and the start of the new gait cycle from both legs. 

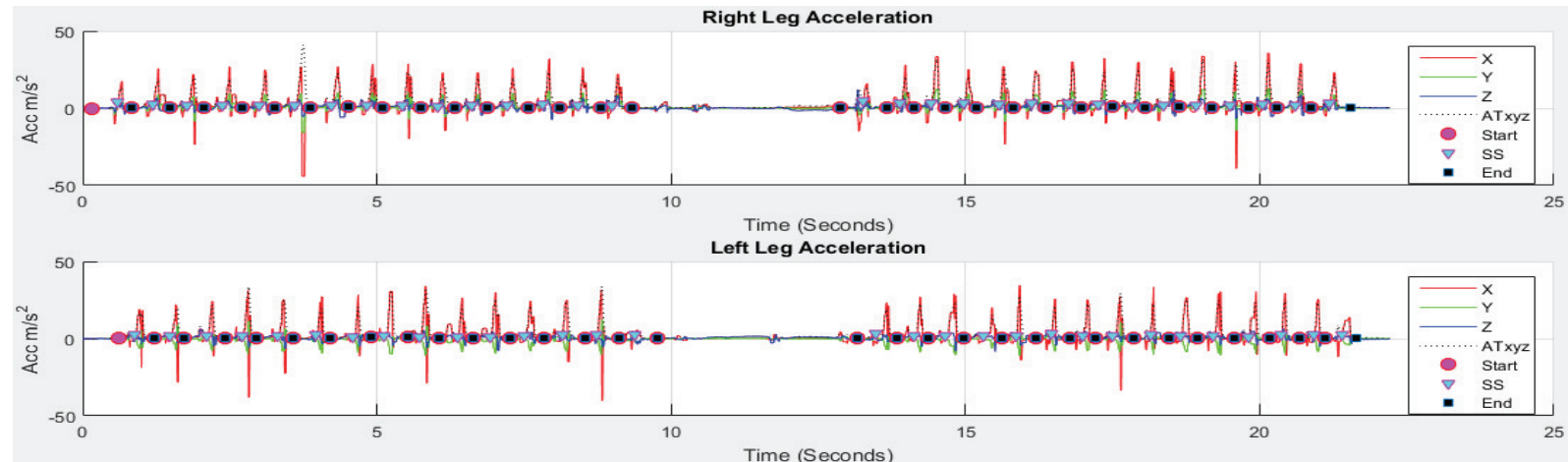

Figure 2. Result of stride, stance and swing event detection

Figure 2 shows detected event of the foot's initial contact to the ground, Start (purple circle), the transition of stance-swing (SS) phase SS (cyan triangle) and the terminal swing End (black square) of gait event information of each stride for both legs where the stance phase information is provided by the difference between Start and SS; and the swing information is the difference between $S S$ and End.

\section{Velocity and distance estimation}

Trapezoidal double integral approach (27) is applied to obtain travelled distance from the user movement using accelerometer data. First integration retrieves the current velocity and then second integration computes velocity and calculates distance travelled. Input data are passed through a high-pass filter to remove the direct component of the acceleration signal. Our measurements of extracting features is validated with Qualysis Motion Capture System (25).

\section{Data and statistical analysis}

Values are obtained for ten spatial-temporal gait parameters separately from the right and left lower limbs include stride length $(\mathrm{m})$, stride time $(\mathrm{s})$, stride velocity $(\mathrm{m} / \mathrm{s})$, step length $(\mathrm{m})$, step time $(\mathrm{s})$, step velocity $(\mathrm{m} / \mathrm{s})$, stance time (s), swing length (m), swing time (s) and swing velocity (m/s). Asymmetry factors SI (10), SR (11), Ia (12), GA (13) and SA (14) are calculated for each parameter using equations (1) to (5) and labelled by corresponding serial no (SL). These are chosen because they are very commonly used approaches of evaluating gait asymmetry (28).

SL1 - SI (10):

$$
\mathrm{SI}(\%)=\frac{\text { RightLeg }- \text { LeftLeg }}{0.5(\text { RightLeg }+ \text { LeftLeg })} * 100
$$


SL3 - Ia (12):

$$
\mathrm{Ia}(\%)=\frac{\text { RighttLeg }- \text { LefttLeg }}{\max (\text { RightLeg,LeftLeg })} * 100
$$

SL4 - GA (3):

$$
\mathrm{GA}=\left|\ln \left\{\frac{\min (\text { RightLeg, LeftLeg })}{\min (\text { RightLeg, LeftLeg })}\right\}\right|
$$

$$
\mathrm{SA}(\%)=
$$

SL5 - SA (14):

$$
\frac{\left(45^{\circ}-\arctan (\text { RightLeg/LeftLeg })\right)}{90^{\circ}} * 100 \%
$$

SL1 is based on percentage assessment of difference between kinematic and kinetic parameters for both legs during walking. $\mathrm{SI}=0$ indicates that there is no asymmetry and $\mathrm{SI} \geq 100 \%$ indicates high asymmetry. SL2 indicates the highest value results asymmetries. SR=100 indicates no asymmetry, SR $>100$ indicates right leg value is higher than left leg and SR $<100$ indicates that left leg value is higher. SL3 is based on kinematic asymmetry of the lower limbs. Ia $=0$ indicates no asymmetry. Ia $= \pm 0$, the higher the value indicates the higher level of asymmetry. SL4 is based on logarithmic transformation of right and left leg's ratio of gait asymmetry. GA=0 and GA=1 denote no asymmetry and highest asymmetry respectively. SL5 is the symmetry angle calculated for the angle of the vector plotted from the right and left values of discrete gait parameters. SA shows absolute value of right and left leg's ratio in percentage. $\mathrm{SA}=0$ indicates no asymmetry and $\mathrm{SA} \geq 100 \%$ indicates asymmetry.

Experimental data are compared to normal distribution using Shapiro Wilk test. Pearson's linear correlation coefficients are calculated. Correlation between experimental results and linear least square regression is analyzed. Although available asymmetry factors SI (10), SR (11), Ia (12), GA (13) and SA (14) provide a numerical indication of the degree of asymmetry they are not easily interpretable to users. These rely on the computation of complex equations as well as knowledge to interpret the results. This may affect the accuracy of use. Therefore, in order to conveniently use quantitative gait asymmetry monitoring, an easy to interpret and affordable gait symmetry visualization tool is required to provide a facility for use in clinic and at home. This paper mainly presents the gait asymmetry visualization to the users, not give the cause of gait asymmetry. Cause of gait asymmetry is an important topic to conduct research in future. 


\section{Visualization of gait}

The visualizations are purposely shown from a single subject as there is no point in showing an aggregate of results from our 20 subjects. Individual visualizations for all are estimated. This section presents four novel gait asymmetry visualization approaches aimed to show the various aspects of gait symmetry analysis and make the results accessible and useful to both patients, for self-directed care, and therapists: 1) Real time dial visualization: this is intended for patient use by providing a spatiotemporal gait information to the patient who can then identify and make attempts to rectify gait asymmetry; 2) Visualization of individual leg time variation: this is intended for therapists assessing gait by giving an overall picture of time asymmetry over a series of strides. In normal human gait the period from the initial contact to pre-swing composes about $60 \%$ of the time and initial swing and terminal swing composes about $40 \%$ of the time in the gait cycle. This visualisation provides therapists the opportunity easily identifies any deviation from this 60:40 split; 3) Visualization of both legs asymmetry: this visualization shows both time and distance for stride and step for both legs. As they are comparing both legs then they would be expected to be as near to equal as possible and any difference is asymmetry; This will also indicate which of the legs is most affected and helps therapists direct attention to the legs with most abnormality; 4) Boxplot-based visualization: this visualization provides an overall summery of the results obtained through the above and therefore can be used to monitor progress with therapy; and 5) Evaluation considering all features: a gait evaluation system using Procrustes and Euclidean distance matrix analysis which offers a simple and easily interpretable assessment of gait with good accuracy and comprehensive features.

\section{1) Real time dial visualization}

Stride, step and swing information is considered for visualization. The stride, stance, swing and step features are extracted. Stance is a stationary phase of a gait cycle and distance travelled in the stationary phase is zero. Initially the maximum (max), minimum $(\min )$, and confidence interval $(C I)$ of each feature are estimated. A circle is drawn from $\theta=0$ to $2 \pi$ of duration of 0.01 using $x=\sin (\theta), y=\cos (\theta)$. The interval is defined $\alpha=50$ and value of each step increment $(\delta)$ is computed by $\delta=(\max -\min ) / \alpha$. Interval angle $\omega$ is estimated using $\omega=\lambda * \pi / \alpha$ with $\lambda=1.25$. Scale is represented from 0 to $\alpha$ using $\gamma=-\lambda * \pi / i * n+\lambda * \pi$, for $n=0$ to $\alpha$. The small scale line is then drawn using $x=\sin (\gamma), y=\cos (\gamma)$. Minimum and maximum values of the scale are the lower $C I$ and upper $C I$ respectively. Indicator line $(\beta)$ is then set with the instantaneous difference between left and right value of the 
feature $(\eta)$ using $\beta=-\omega^{*}(\eta-\min ) / \delta+\lambda * \pi$. The indicator line is drawn from 0 to $\beta$. A gradient is drawn colors to make it colorful looking. Instantaneous feature value is displayed at the bottom of each dial with seven segment display. The same procedure is followed for displaying instantaneous distance and time from stride, step and swing information.

When the app is run for the first time, there is an option to input a number of last performed strides. By default, the value is set as 30 . It will then detect phases and display corresponding information on to dial. Every time it starts, it will restore the last calculated $C I$ for scaling and it will update automatically after each 30 or specified numbers of strides. There is an option to change the scaling factor according to SI (10), SR (11), Ia (12), GA (13) and SA (14) format.

\section{2) Visualization of individual leg time variation}

Each stride is composed of stance and swing phases. Stride time is composed of stance time and swing time. To visualize the individual legs variation, the maximum and minimum values are estimated of each feature. An outline rectangle is drawn using blue color towards vertical line to represent right of the first stride value with aspect ratio of the maximum value. A cyan color rectangle of stance time is drawn on that stride rectangle and a yellow color rectangle of swing time is drawn at the top of the stance rectangle. All rectangles are followed the aspect ratio with the maximum value. This process is conducted for all stored strides.

\section{3) Visualization of both legs asymmetry}

Each stride and step feature has distance and time information. Initially the maximum and minimum of features are calculated. A red color rectangle is drawn which height is the first right stride distance with aspect ratio of the maximum value. A blue rectangle is drawn which height is the left stride distance at the side of right rectangle. We follow this procedure for both strides and step asymmetry visualization of all stored strides considering the aspect ratio with the maximum value.

\section{4) Boxplot-based visualization}

The median, upper-quartile, lower quartile and whisker values are estimated from features and plot a Boxplot. This is a simple representation of descriptive statistics to understand each features distribution, non-normal/unusual level, outliers, symmetry and overall gait asymmetry information. 


\section{5) Evaluation considering all features}

In order to quantify and compare gait, a common procedure is to align the shape developed from the extracted gait features both in time and length. The eight extracted gait features (stride length, stride time, stride velocity, step length, step time, step velocity, stance time, swing length, swing time and swing velocity) from right and left legs are transferred in the Cartesian coordinate shown in Figure 3. The $x$ and $y$ axes represent the features of the right and left legs with the dimensionless numbers respectively. Eight features of all individual subjects are plotted and each of these points is notionally joined together to represent a shape. Procrustes analysis (a method of statistical analysis used to analyse the distribution of a set of shapes) describes curve shape and shape change in a mathematical and statistical framework, independently of time and size factors. A standard normal mean gait shape (NMGS) is estimated using Procrustes superimposition. Each gait shape is then converted to find the best match with the NMGS. Euclidean distance matrix analysis (EDMA) is used to analyze the influence of each feature. The degree of abnormality is then estimated for individual features and the result is plotted to visualize the feature (29).
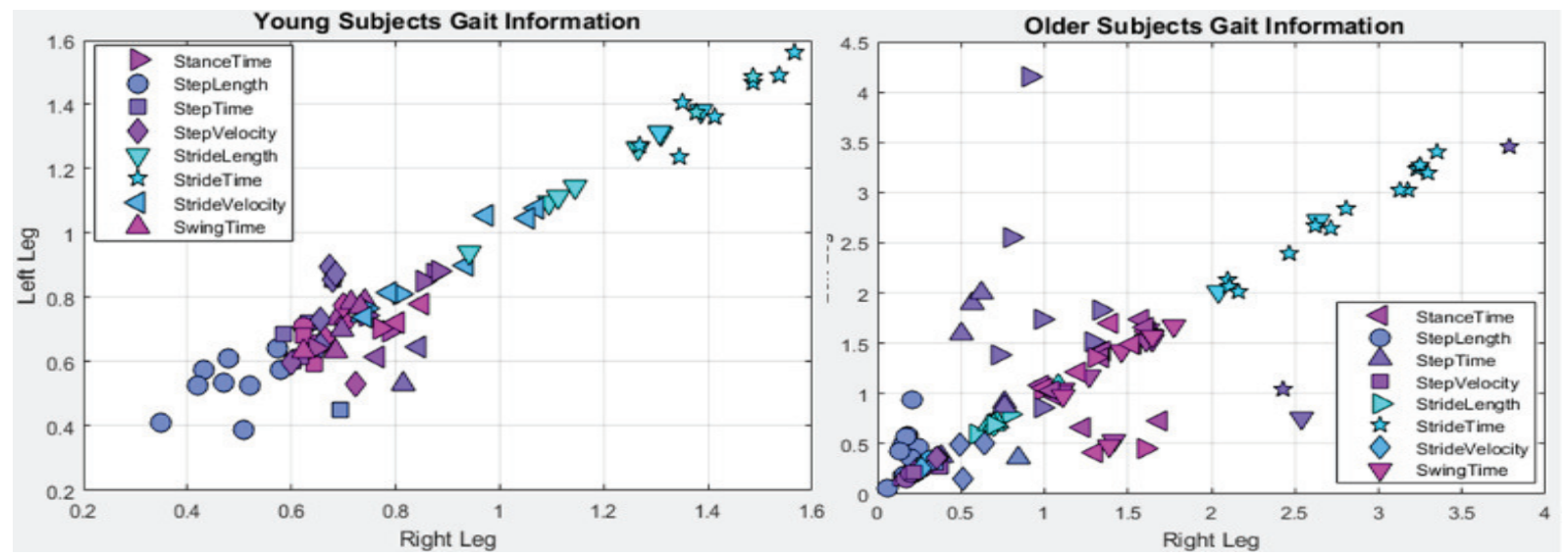

Figure 3: Gait features from young and older adults

\section{Results}

Preliminary experimental results from a subject: automatic gait asymmetry features are extracted based on accelerometer and gyroscope data collected from both feet.

\section{Gait features information}

The total time taken to travel 33.38 meters is 22.21 seconds. The estimated legs travelled distances are 33.35 meters and 32.87 meters with accuracy of $99.92 \%$ and $98.48 \%$. Actual and estimated distances are very close. Accuracy of 
stride and step event detection is $100 \%$. Table 1 shows the average gait variability and quantifying gait asymmetry using five techniques.

\begin{tabular}{|c|c|c|c|c|c|c|c|c|c|}
\hline & \multicolumn{2}{|c|}{ Right Leg } & \multicolumn{2}{c|}{ Left Leg } & SL1 & SL2 & SL3 & SL4 & SL5 \\
\hline Gait Features & Mean & $\mathbf{9 5 \%}$ CI & Mean & $\mathbf{9 5 \%}$ CI & SI & SR & Ia & GA & SA \\
\hline Stride Length (m) & 1.112 & 0.225 & 1.096 & 0.230 & 1.45 & 101.46 & -1.44 & 0.01 & 0.46 \\
\hline Stride Time (s) & 0.595 & 0.027 & 0.588 & 0.026 & 1.13 & 101.13 & -1.12 & 0.01 & 0.36 \\
\hline Stride Velocity (m/s) & 1.823 & 0.308 & 1.855 & 0.371 & -1.73 & 98.29 & 1.71 & 0.02 & -0.55 \\
\hline Step length (m) & 0.507 & 0.041 & 0.387 & 0.043 & 26.68 & 130.79 & -23.54 & 0.27 & 8.44 \\
\hline Step time(s) & 0.258 & 0.028 & 0.337 & 0.027 & -26.58 & 76.53 & 23.47 & 0.27 & -8.41 \\
\hline Step Velocity (m/s) & 2.185 & 0.337 & 1.256 & 0.223 & 53.98 & 173.94 & -42.51 & 0.55 & 16.78 \\
\hline Stance Time (s) & 0.315 & 0.018 & 0.278 & 0.021 & 12.36 & 113.17 & -11.64 & 0.12 & 3.93 \\
\hline Swing Length (m) & 1.009 & 0.202 & 0.990 & 0.207 & 1.88 & 101.90 & -1.86 & 0.02 & 0.60 \\
\hline Swing Time (s) & 0.280 & 0.019 & 0.310 & 0.021 & -10.18 & 90.31 & 9.69 & 0.10 & -3.24 \\
\hline Swing Velocity (m/s) & 1.729 & 0.248 & 1.537 & 0.248 & 11.79 & 112.53 & -11.13 & 0.12 & 3.75 \\
\hline
\end{tabular}

\section{Table 1: Gait variability and asymmetry factors (SI, SR, Ia, GA and SA)}

Table 1 shows that the mean stride lengths and times of both legs are very close. However, high asymmetry is found in step length, time and velocity. Estimated asymmetry factors show numerical values that indicate differences both between the features and between the indicators. The lowest gait asymmetry is observed during the stride phase and the highest is found during the step event. Using the Shapiro-Wilk test, most of the parameters show normal distribution for 20 participants. Additionally, the lowest $C I$ are observed for most of the parameters indicating consistent data.

Analysis of Pearson linear correlation coefficients between SL1, SL2, SL3, SL4 and SL5 factors indicates a very strong association $(\mathrm{p}<0.001)$ for most of the cases excluding SL4. As such, it is more useful to analyze the compatibility of the results for individual factors in the assessment of the symmetry of the factors indicating high symmetry. Coefficient ordered rank also agrees for most of the cases. The linear least square regressions show very high correlations. It is important for clinical practice to evaluate the impact of individual factors resulting high gait symmetry and the interpretation of these numerical values provide limited information. Therefore, a visual representation of these values with interpretation would provide much more user friendly information.

\section{Gait visualization}

Next, the results of the four gait asymmetry visualizations are shown.

\section{1) Real time dial visualization}

Figure 4 demonstrates spatiotemporal measurements in a dial fashion taken from one subject. Both legs should theoretically give identical results and therefore perfect asymmetry should give dial indicator readings of zero. The 
first dial is an asymmetry display for stride length and time comparing both legs. The second dial displays the real time measurement of step length and time. It is noted that there is a difference in the level of asymmetry. The third dial shows the swing phase distance and time. Similarly, there is little asymmetry between two legs. The scales on all three dials represent the $C I$ s and the pointer represents the instantaneous real time difference between two legs. For example, in this case although the dials for stride and swing show near perfect symmetry, measurements relating to step are not. Step measurement entails information on distance and time. The distance dial shows that right leg is travelling longer $(0.51 \mathrm{~m})$ than left leg $(0.39 \mathrm{~m})$. The patient therefore needs to shorten the distance travelled by the right leg and/or make the left step longer. Time dial demonstrates the right leg travels the longer distance in a shorter time $(0.26 \mathrm{~s})$ compared to the left leg $(0.34 \mathrm{~s})$. Digital number below the dial is showing the absolute measure for all three markers.

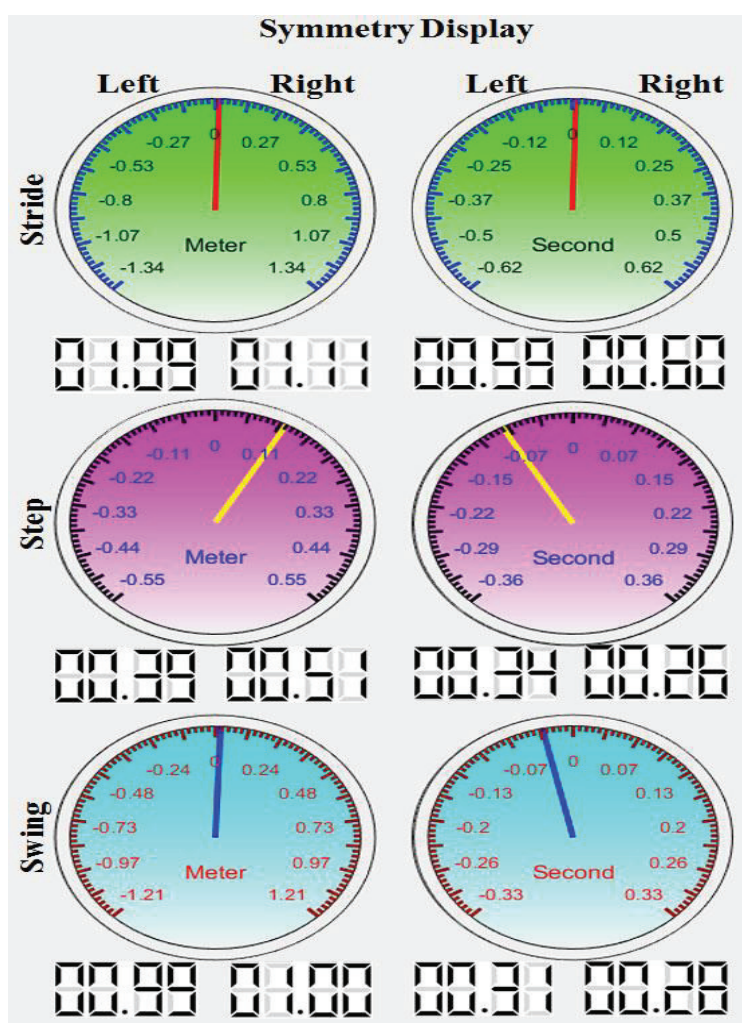

Figure 4. Real time gait asymmetry visualization

\section{2) Visualization of individual leg time variation}

30 strides are performed and time of stride, stance and swing phases is presented in Figure 5 where each bar shows the stride time. 

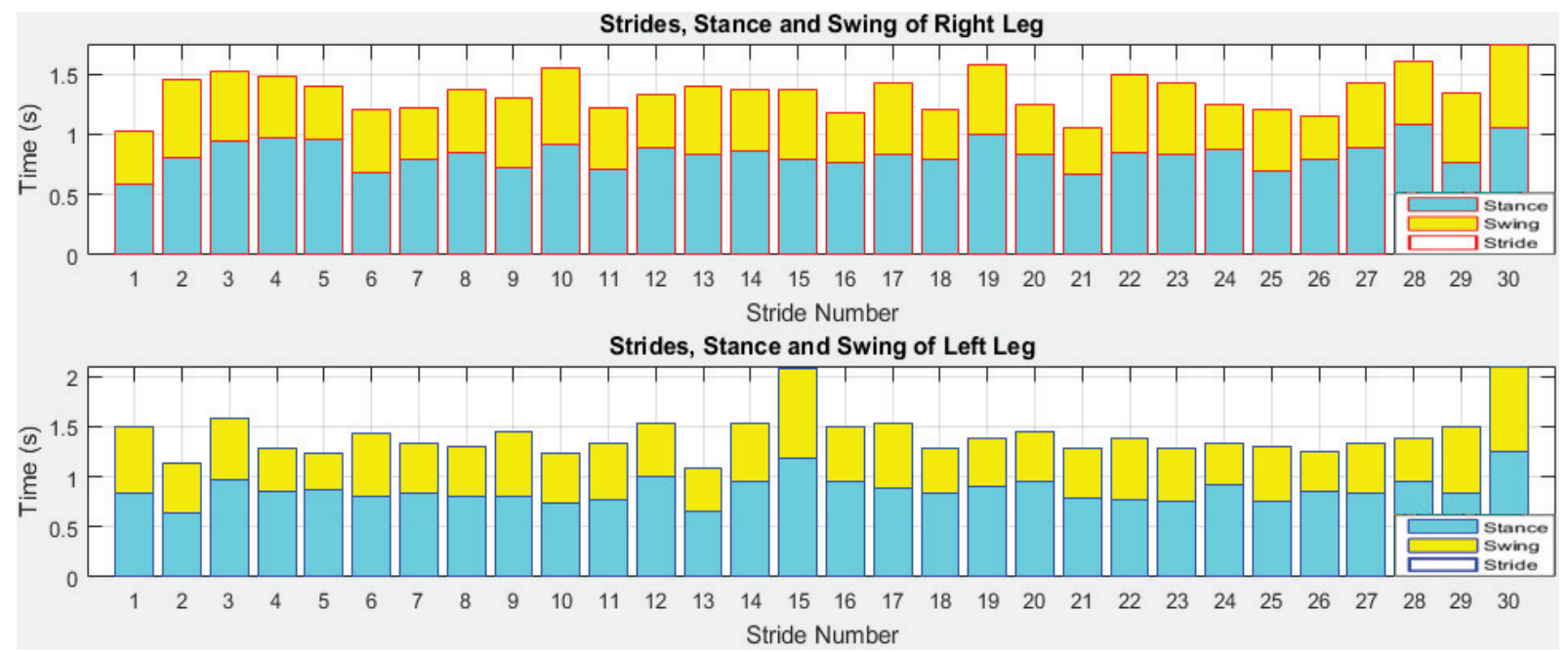

Figure 5. Time of stride, stance and swing phases from right and left legs

Cyan and yellow colors represent the time of stance and swing phases respectively. There is a small variation of stance and swing phase timing. This visualization clearly represents the variability of stance and swing phases in each stride of the legs. The ratio of stance and swing is found closest to the $60: 40 \%$ split for average stride, stance and swing information (Figure 5).

\section{3) Visualization of both legs asymmetry}

In this visualization the stride and step asymmetry information for both time and distance from both legs are presented in Figure 6. We observe that while there is good symmetry in the stride there is strong variation in the step phases. 